\title{
Increased expression of C5a receptor (CD88) mRNA in canine mammary tumors.
}

\begin{abstract}
Mammary tumors are among the most common neoplastic conditions in dogs, and there is evidence that inflammation plays a role in the development of some tumor types in dogs. The complement system is a major participant in the inflammatory process and the complement activation component, $\mathrm{C} 5 \mathrm{a}$, is a potent inflammatory peptide. This study investigated the mRNA expression of the major receptor for C5a (C5aR; CD88) in histopathological samples of canine mammary tumors by quantitative reverse transcriptase-polymerase chain reaction (qRT-PCR) using canine-specific primers for CD88. A total of seven canine mammary tumors (four malignant carcinomas, two benign mixed mammary tumors, and one myoepithelioma) and eight normal mammary glands were analysed. All the tumor samples expressed low levels of CD88 mRNA, while none of the normal mammary tissues showed any detectable expression. These preliminary results suggest that C5a-CD88 interaction may play a contributory role in the inflammatory response associated with mammary tumor development in dogs. Further studies investigating the mechanisms behind complement activation and C5a receptor expression in canine mammary tumors are warranted
\end{abstract}

Keyword: Canine mammary tumor; Complement factor C5a; Polymerase chain reaction (PCR); C5a receptor. 